\title{
Characteristics of Mg-doped and In-Mg co-doped p-type GaN epitaxial layers grown by metal organic chemical vapour deposition
}

\author{
S J Chung ${ }^{1}$, M Senthil Kumar ${ }^{2}$, Y S Lee ${ }^{1}$, E-K Suh ${ }^{1,4}$ and M H An ${ }^{3}$ \\ ${ }^{1}$ Semiconductor Physics Research Center, Chonbuk National University, Chonju 561-756, \\ Republic of Korea \\ ${ }^{2}$ Electronic Materials Division, National Physical Laboratory, New Delhi 110 012, India \\ ${ }^{3}$ Division of Mechanical and Automotive Engineering, Chonnam National University, Yosu 550-749, \\ Republic of Korea
}

E-mail: eksuh@chonbuk.ac.kr

\begin{abstract}
Mg-doped and In-Mg co-doped p-type GaN epilayers were grown using the metal organic chemical vapour deposition technique. The effect of In co-doping on the physical properties of p-GaN layer was examined by high resolution x-ray diffraction (HRXRD), transmission electron microscopy (TEM), Hall effect, photoluminescence (PL) and persistent photoconductivity (PPC) at room temperature. An improved crystalline quality and a reduction in threading dislocation density are evidenced upon In doping in $\mathrm{p}-\mathrm{GaN}$ from HRXRD and TEM images. Hole conductivity, mobility and carrier density also significantly improved by In co-doping. PL studies of the In-Mg co-doped sample revealed that the peak position is blue shifted to $3.2 \mathrm{eV}$ from $2.95 \mathrm{eV}$ of conventional $\mathrm{p}-\mathrm{GaN}$ and the PL intensity is increased by about $25 \%$. In addition, In co-doping significantly reduced the PPC effect in p-type GaN layers. The improved electrical and optical properties are believed to be associated with the active participation of isolated $\mathrm{Mg}$ impurities.
\end{abstract}

\section{Introduction}

Following the tremendous progress in the studies of group III-nitrides, high efficiency blue/green light emitting diodes and laser diodes have been successfully commercialized [1,2]. Realization of p-type $\mathrm{GaN}$ by $\mathrm{Mg}$ doping has been the key achievement in the development of $\mathrm{GaN}$ based optoelectronic devices. Despite the steady progress, many properties of the nitride material system remain to be understood well. In particular, the characteristics of $\mathrm{Mg}$ dopants in $\mathrm{GaN}$ are still not clear. Until now, Mg is the only successful dopant to have achieved reasonable hole conduction for realizing practical devices. Generally, the doped $\mathrm{Mg}$ concentration is about two orders higher than the obtained hole densities. $\mathrm{Mg}$ doping can generate numerous native defects, causing severe self-compensation effects that degrade the film properties $[3,4]$. Isoelectronic In doping has been found to be an efficient technique to improve the optical and electrical properties of $\mathrm{GaN}$ based nitride epilayers and quantum structures [5-9]. However, there have been very few reports on the effect of In co-doping in p-type $\mathrm{GaN}$ layers $[10,11]$. The purpose of this study is to investigate the effect of In co-doping on the physical properties of p-type GaN layers grown by metal organic chemical vapour deposition (MOCVD).

The persistent photoconductivity (PPC) technique has been effectively used for studying the optical properties of group III-nitrides. There are several reports available on the PPC of undoped, n-type and p-type GaN, InGaN, Si-doped InGaN and $\mathrm{AlGaN}$ [12-14]. In these samples, the PC decay and time constant have been extensively studied. However, the PPC study of In co-doped p-GaN epitaxial layers has 
not yet been reported. We have observed that In co-doping decreases the PPC effect in p-type GaN and also increases the photocurrent. In this paper, we report a significant improvement of the structural, electrical and optical properties of p-type GaN layers by In co-doping.

\section{Experiment}

Mg-doped and In-Mg co-doped p-GaN epilayers were grown on $c$-plane sapphire substrates using the MOCVD technique. Trimethylgallium (TMGa), trimethylindium(TMIn), $\mathrm{CP}_{2} \mathrm{Mg}$ and ammonia were used as precursors for the $\mathrm{p}-\mathrm{GaN}$ growth. Firstly, the sapphire substrate was thermally cleaned at $1150^{\circ} \mathrm{C}$ in hydrogen ambient in a reactor. Then, a $25 \mathrm{~nm}$ nucleation layer was grown at $530^{\circ} \mathrm{C}$ followed by a $300 \mathrm{~nm}$ thick undoped $\mathrm{GaN}$ layer grown at $1100^{\circ} \mathrm{C}$. Finally, the $\mathrm{Mg}$ and $\mathrm{In}-\mathrm{Mg}$ doped p-type $\mathrm{GaN}$ layers were grown at $1050^{\circ} \mathrm{C}$ for a thickness of $1.02 \mu \mathrm{m}$ and $0.9 \mu \mathrm{m}$, respectively. TMIn and $\mathrm{CP}_{2} \mathrm{Mg}$ flow rates were fixed to be $0.4 \mu \mathrm{mol} \mathrm{min}{ }^{-1}$ and $38 \mu \mathrm{mol} \mathrm{min}^{-1}$, respectively. After growth, the doped $\mathrm{GaN}$ layers were thermally annealed by RTA at $940^{\circ} \mathrm{C}$ for $40 \mathrm{~s}$ to activate the dopant. The $\mathrm{Mg}$ activated samples were then characterized using high resolution X-ray diffraction (HRXRD), transmission electron microscopy (TEM), Hall effect, photoluminescence (PL) and PPC techniques at room temperature.

A PL excitation source of $\mathrm{He}-\mathrm{Cd}$ laser of $325 \mathrm{~nm}$ with a power density of $20 \mathrm{~mW} \mathrm{~cm}^{-2}$ was used. For the PPC measurement, a halogen lamp of $1000 \mathrm{~W}$ and a Shimadzu AQV50 spectrometer were employed to make the illumination source. The GaN layer was excited by a light of energy equal to the band energy of GaN emerging from the spectrometer. To ensure the true dark current level, the samples were kept at room temperature for $6 \mathrm{~h}$ before turning on the excitation light source. After $2000 \mathrm{~s}$ of electric bias and illumination, the light was turned off to carry out the current decay measurement.

\section{Results and discussion}

The x-ray rocking curves of the $\mathrm{Mg}$-doped and $\mathrm{In}-\mathrm{Mg}$ co-doped p-type GaN epilayers measured along the symmetric $\left(\begin{array}{lll}0 & 0 & 2\end{array}\right)$ and asymmetric $\left(\begin{array}{lll}1 & 0 & 2\end{array}\right)$ planes are shown in figures $1(a)$ and $(b)$. The $(002)$ plane FWHM values of 277 arcsec and 269 arcsec and the (102) plane FWHM values of 613 arcsec and 434 arcsec were obtained for the Mg-doped and In-Mg co-doped p-GaN layers, respectively. The high intensity of the $\mathrm{x}$-ray rocking curve in both reflections shows the good crystalline nature of the In-Mg co-doped GaN sample. The very low FWHM along the ( $\left.\begin{array}{lll}1 & 0 & 2\end{array}\right)$ asymmetric plane diffraction of the $\mathrm{In}-\mathrm{Mg}$ co-doped $\mathrm{GaN}$ layer clearly indicates a reduction in edge threading dislocation (TD) density upon In co-doping. The cross-sectional TEM images of Mg-doped and In-Mg co-doped p-GaN layers are also presented in figure 2. As seen, it is observed that the TD density is decreased upon In co-doping in the Mg-GaN samples. It has been previously reported that In co-doping can lead to pinning of TD and annihilation of related native defects in the GaN layer thereby improving the crystalline quality [7]. Surface pit densities for the $\mathrm{Mg}$-doped and $\mathrm{In}-\mathrm{Mg}$ co-doped $\mathrm{GaN}$ layers were

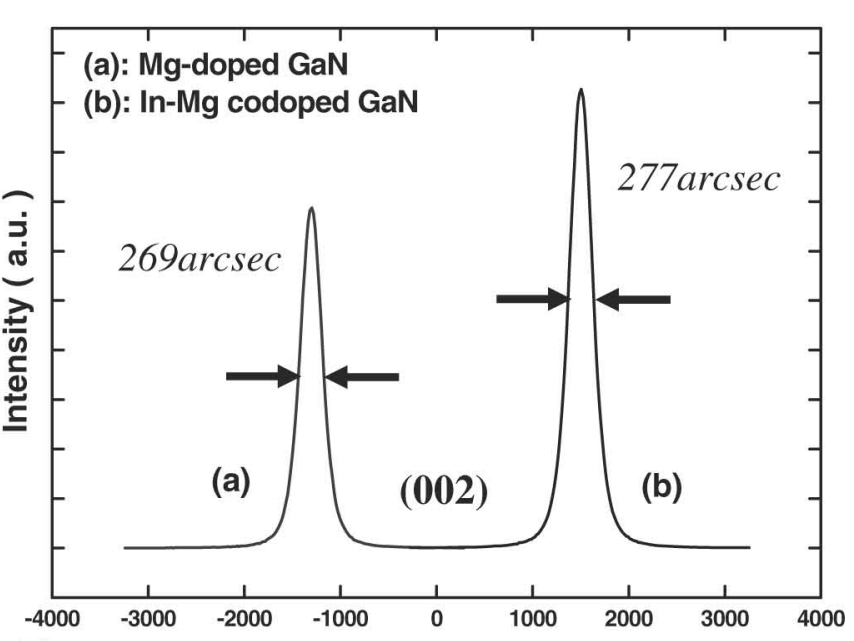

(a) HRXRD rocking curve (arcsec)

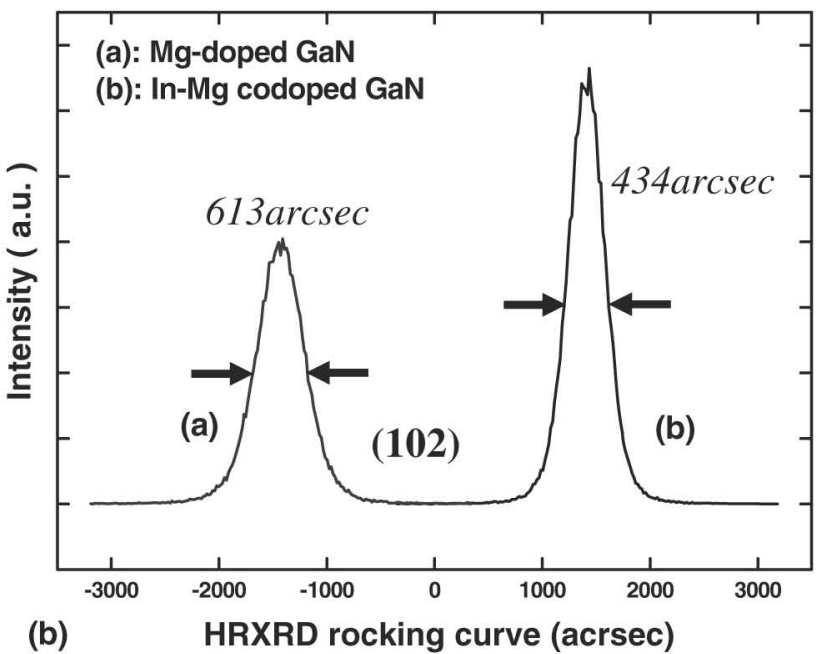

Figure 1. HRXRD rocking curves of $\mathrm{Mg}$-doped and In-Mg co-doped GaN along (a) symmetric ( 002$)$ and $(b)$ asymmetric diffraction planes.

measured, respectively, as $5.12 \mathrm{E} 09 \mathrm{~cm}^{-2}$ and $4.75 \mathrm{E} 09 \mathrm{~cm}^{-2}$ using atomic force microscopy, which is an indication of TD reduction in the In co-doped p-GaN layer. The electrical characteristics of $\mathrm{Mg}$-doped and $\mathrm{In}-\mathrm{Mg}$ co-doped p-type GaN layers measured by the room temperature Hall effect are given in table 1 . In co-doping significantly improved the electrical properties of the p-type GaN layers. The hole conductivity, mobility and carrier density values of the co-doped samples considerably increased compared with the conventionally grown p-type GaN layer. This result implies that the reduction in TD density and associated defects has an impact on the electrical properties of the In co-doped p-GaN layer that might be attributed to the reduction of self-compensation centres. On the other hand, it is also likely that In co-doping enhances the doping efficiency of the $\mathrm{Mg}$ dopant to provide more carriers with less impurities, thus improving the hole mobility due to reduced impurity scattering. Further study is underway to clarify this point.

The room temperature PL spectra of Mg-doped and In-Mg co-doped p-GaN layers are compared in figure 3. In general, the band-edge PL emission of unintentionally doped $\mathrm{GaN}$ is 


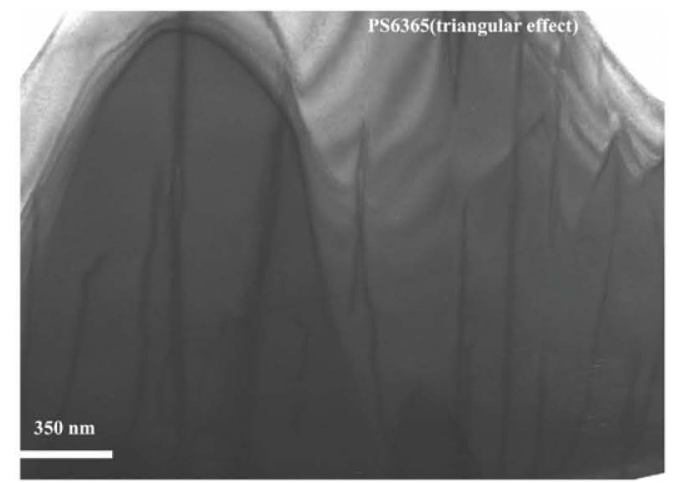

(a)

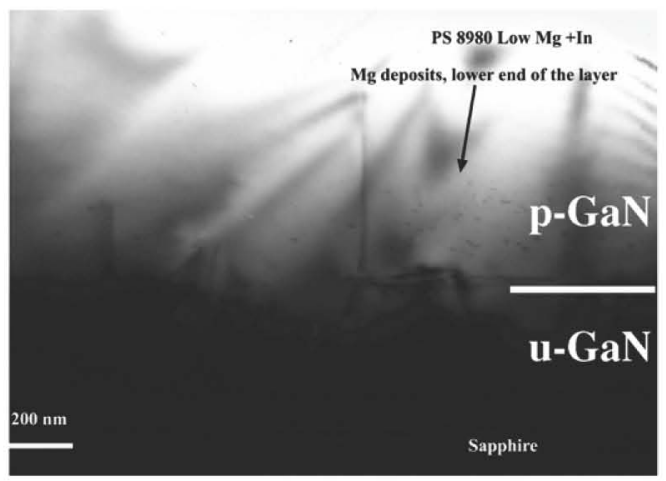

(b)

Figure 2. Cross-sectional TEM images of (a) Mg-doped and (b) In-Mg co-doped GaN.

Table 1. Room temperature Hall effect measurements of Mg-doped and In-Mg co-doped p-type GaN layers.

\begin{tabular}{llcl}
\hline p-GaN & $\begin{array}{l}\text { Sheet } \\
\text { resistivity } \\
\text { samples }\end{array}$ & $\begin{array}{l}\text { Mobility } \\
(\Omega / \square)\end{array}$ & $\begin{array}{l}\text { Sheet hole } \\
\left(\mathrm{cm}^{2} \mathrm{~V}^{-1} \mathrm{~s}^{-1}\right)\end{array}$ \\
\hline density $\left(\mathrm{cm}^{-2}\right)$
\end{tabular}

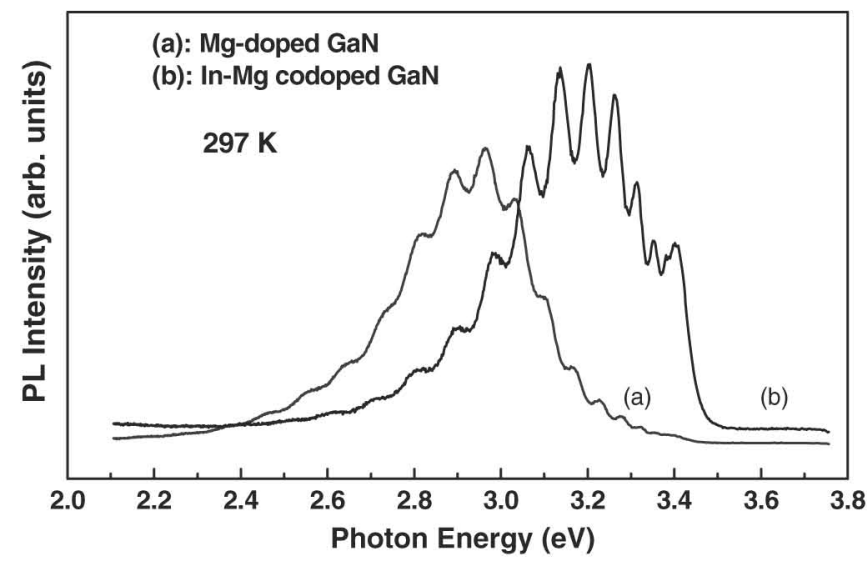

Figure 3. Room temperature PL spectra of (a) Mg-doped and (b) In-Mg co-doped GaN.

observed at $3.4 \mathrm{eV}$. But, for the Mg-doped GaN layer, the main PL peak is broadened and red shifted to $2.95 \mathrm{eV}$ as shown in figure 3. Sheu et al have reported a PL emission of $2.95 \mathrm{eV}$ for Mg-doped GaN and attributed the PL transition to a donor-toacceptor (DAP) pair [15]. They have suggested that the DAP transition line is caused by a $\mathrm{Mg}$ related deep level at about $510 \mathrm{meV}$ above the valence band, whereas, Kaufmann et al observed a PL peak at $2.8 \mathrm{eV}$ for $\mathrm{Mg}$-doped $\mathrm{GaN}$ and suggested a DAP transition from the deep donor $(430 \mathrm{eV})$ level to an isolated $\mathrm{Mg}_{\mathrm{Ga}}$ acceptor level [16]. As In atoms are co-doped in our p-GaN sample, the PL peak is blue shifted to $3.2 \mathrm{eV}$ with respect to the $\mathrm{Mg}$-doped $\mathrm{GaN}$ peak and the PL intensity is also enhanced by about $25 \%$. Chang et al have observed a similar blue shifted peak at $3.1 \mathrm{eV}$ for the In-Mg co-doped p-GaN layer that complements our result [11]. For Mg-doped p-GaN, they obtained the PL peak at $2.8 \mathrm{eV}$ and attributed it to the transition either from the shallow donor to the $\mathrm{Mg}$ related deep level or

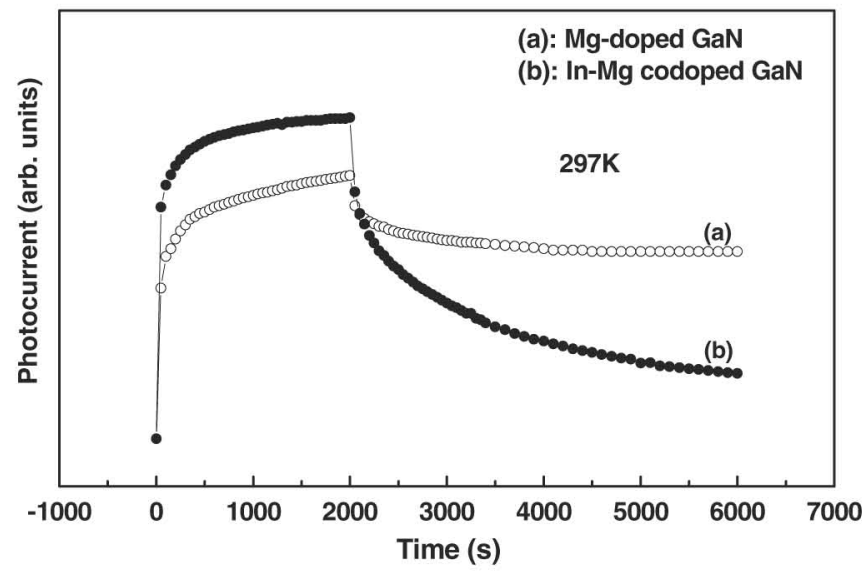

Figure 4. Room temperature PPC spectra of (a) Mg-doped and (b) In-Mg co-doped GaN.

from the deep donor to the common $\mathrm{Mg}$ acceptor level. The peak observed at $3.1 \mathrm{eV}$ for the $\mathrm{In}-\mathrm{Mg}$ co-doped sample was explained based on the transition from the conduction band to the isolated $\mathrm{Mg}_{\mathrm{Ga}}$ level.

Based on theoretical calculations, Neugebauer and Vand de Walle stated that the only negative defect with a relevant concentration in $\mathrm{p}-\mathrm{GaN}$ is a nitrogen vacancy $\left(\mathrm{V}_{\mathrm{N}}\right)$ and its concentration is nearly the same as the $\mathrm{Mg}$ concentration at commonly used MOCVD growth temperatures $[17,18]$. The oppositely charged $\mathrm{V}_{\mathrm{N}}$ and the $\mathrm{Mg}$ acceptor tend to form a $\mathrm{Mg}_{\mathrm{Ga}}-\mathrm{V}_{\mathrm{N}}$ complex acting as compensation centres. Hence, we assign the $2.95 \mathrm{eV}$ PL peak obtained for the Mg-doped $\mathrm{GaN}$ to the transition between the shallow donor and the $\mathrm{Mg}_{\mathrm{Ga}}-\mathrm{V}_{\mathrm{N}}$ complex related deep level. For an In-containing alloy, it is well documented that a small addition of In atoms in the growth suddenly decreases the $\mathrm{Ga}$ incorporation efficiency leading to a higher effective V/III ratio at the growth surface $[9,19]$. This results in a decrease in $\mathrm{V}_{\mathrm{N}}$ in the solid and will increase the $\mathrm{Mg}_{\mathrm{Ga}}$ concentration. Hence, the increased $\mathrm{Mg}_{\mathrm{Ga}}$ might contribute to the strong PL intensity around $3.2 \mathrm{eV}$ for the In-Mg co-doped $\mathrm{p}-\mathrm{GaN}$ layers.

To further explore the optical characteristics of In co-doped $\mathrm{p}-\mathrm{GaN}$, we have performed PPC measurements on both $\mathrm{Mg}$-doped and $\mathrm{In}-\mathrm{Mg}$ co-doped $\mathrm{GaN}$ layers. Figure 4 shows the comparison of PPC spectra of these samples. As seen, a strong PPC effect is observed in our Mg-doped p-GaN 
sample. However, In-Mg co-doping has clearly decreased the PPC effect in the p-type GaN and also increased the photocurrent, which is an indication of improved epilayer quality by In co-doping. Many authors have observed metastability and PPC effects in Mg-doped p-type GaN epilayers. A significant paper by $\mathrm{Li}$ et al reported an anomalously slow thermal equilibrium process for the dark carrier concentration at low temperatures in $\mathrm{Mg}$-doped $\mathrm{p}-\mathrm{GaN}$. Based on those results, they proposed that neutral impurities in p-type GaN have two different states, a metastable and a stable state [20]. These two states probably correspond to two different local configurations of neutral impurities and there is a potential barrier between the metastable and the stable states of neutral impurities. The physical origin responsible for these behaviours was considered to be isolated $\mathrm{Mg}$ acceptors and associated lattice relaxation. However, as the growth environment of the MOCVD technique includes hydrogen, it could also be associated with the presence of isolated $\mathrm{H}$ impurities or $\mathrm{Mg}-\mathrm{H}$ complexes since the presence of $\mathrm{H}$ can lead to a metastability behaviour. However, later, the same group reported the comparative results of the metastable nature of both MOCVD and MBE grown Mg-doped p-type $\mathrm{GaN}$ concluding that the lattice relaxation associated with $\mathrm{Mg}$ impurities causes the metastable nature in both cases [21]. With this background, the reduced PPC effect in the In-Mg co-doped p-type GaN layers might be associated with the change in local configurations of $\mathrm{Mg}$ impurity by In co-doping but demands further investigations to clearly understand this phenomenon.

\section{Summary}

The characteristics of MOCVD grown Mg-doped and In-Mg co-doped p-type GaN layers have been studied. In-Mg co-doping for p-type GaN layers is found to improve the crystalline quality with reduced TD density as characterized by HRXRD and TEM. Hall measurements showed high hole conductivity, mobility and carrier density as a result of In co-doping. Room temperature PL intensity significantly increased and the peak position shifted to $3.2 \mathrm{eV}$ from $2.95 \mathrm{eV}$ of the conventional p-GaN layer. Also, the In co-doping reduced the PPC effect and enhanced the photocurrent of p-GaN layers. The improved physical properties of the In co-doped p-type GaN layer are attributed to the high crystalline quality and to the active participation of incorporated $\mathrm{Mg}$ impurities.

\section{Acknowledgments}

This work was supported by the Korea Research Foundation Grant funded by the Korea Government (KRF-2008-005J00301).

\section{References}

[1] Nakamura S, Senoh M, Iwasa N and Nagahama S 1995 Japan. J. Appl. Phys. 34 L797

[2] Nakamura S, Senoh M, Nagahama S, Iwasa N, Yamada T, Matsushita T, Kiyoku H and Sugimoto H 1996 Japan. J. Appl. Phys. 35 L74

[3] Gotz W, Johnson N M and Bour D P 1996 Appl. Phys. Lett. 683470

[4] Chadi D J 1997 Appl. Phys. Lett. 712970

[5] Yamaguchi S, Kariya S, Nitta S, Kashima M. Kosaki M, Yukawa Y, Amano H and Akasaki I $2000 \mathrm{~J}$. Cryst. Growth 221327

[6] Yamaguchi S, Kariya M, Nitta S, Amano H and Akasaki I 2000 Japan. J. Appl. Phys. 392385

[7] Yamaguchi S, Kariya M, Kashima T, Nitta S, Kosaki M, Yukawa Y, Amano H and Akasaki I 2001 Phys. Rev. B 64035318

[8] Cho H K, Kim C S, Hong Y K, Kim Y-W, Hong C-H, Suh E-K and Lee H J 2001 Phys. Status Solidi b 228231

[9] Chung H M, Chuang W C, Pan Y C, Tsai C C, Lee M C, Chen W H, Chen W K, Chiang C I, Lin C H and Chang H 2000 Appl. Phys. Lett. 76897

[10] Chang F C, Shen K C, Chung H M, Lee M C, Chen W H and Chen W K 2002 Chin. J. Phys. 40637

[11] Chang F-C, Chou W-C, Chen W-H, Lee M-C, Chen W-K and Huang H-Y 2005 Japan. J. Appl. Phys. 447504

[12] Hirsch M T, Wolk J A, Walukiewicz W and Haller E E 1997 Appl. Phys. Lett. 711098

[13] Qiu C H and Pankove J I 1997 Appl. Phys. Lett. 701983

[14] Chung S J, Jeong M S, Cha O H, Hong C-H, Suh E-K and Lee H J 2000 Appl. Phys. Lett. 761021

[15] Sheu J K, Su Y K, Chi G C, Pong B J, Chen C Y, Huang C N and Chen W C 1998 J. Appl. Phys. 844590

[16] Kaufmann U, Kunzer M, Maier M, Obloh H, Ramakrishnan A, Santic B and Schlotter P 1998 Appl. Phys. Lett. 721326

[17] Neugebauer J and Van de Walle C C 1994 Phys. Rev. B $\mathbf{5 0} 8067$

[18] Neugebauer J and Van de Walle C G 1996 Appl. Phys. Lett. 681829

[19] Ou J, Chen W K, Lin H C, Pan Y C and Lee M C 1998 Japan. J. Appl. Phys. 37 L633

[20] Johnson C, Lin J Y, Jing H X, Asif Khan M and Sun C J 1996 Appl. Phys. Lett. 681808

[21] Li J Z, Lin J Y, Jiang H X, Salvador A, Botchkarev A and Morkoc H 1996 Appl. Phys. Lett. 691474 